\title{
Modeling Surface Hydrology Concepts with Endurance and Perdurance
}

\author{
Chen-Chieh Feng ${ }^{1}$, Thomas Bittner ${ }^{2}$, and Douglas M. Flewelling ${ }^{1}$ \\ ${ }^{1}$ Department of Geography, University at Buffalo \\ 105 Wilkeson Quad, Amherst, NY 14261, USA \\ \{cfeng, dougf\}@geog.buffalo.edu \\ ${ }^{2}$ Institute for Formal Ontology and Medical Information Sciences, University of Leipzig \\ Härtelstraße 16-18, 04107 Leipzig, Germany \\ thomas.bittner@ifomis.uni-leipzig.de
}

\begin{abstract}
Integration of GIS and hydrologic models has been a common approach for monitoring our ever-changing hydrologic system. One important issue in adapting such an approach is to ensure the right correspondence of data across databases. To reach this goal, it is necessary to develop a description of the surface hydrology concepts that is internally consistent and semantically rich. In this paper, we apply the notions of endurance and perdurance to model the semantics of hydrologic processes in surface hydrology. Three hydrologic models were examined to identify concepts used in surface hydrology. The paper demonstrates the usefulness of applying the notions of endurance and perdurance to surface hydrology. The result is a set of primitive entities, aggregate entities, and relations between these entities that are necessary to cover surface hydrology concepts.
\end{abstract}

\section{Introduction}

Modeling the semantics of spatial dynamics has always been a key issue in advancing geographic information science. By incorporating the temporal dimension and the concept of change into a geographic information database, reality can be portrayed more faithfully than through the lenses of cartographic constructs [7]. Capturing such semantics is especially desirable in an interoperable environment, because the identification of suitable geographic information usually cannot be done with the help of human experts. While methods for the identification of such geographic information are available [5, 13], a complete description of such geographic information is still needed for those methods to be useful.

The objective of this research is to improve the usability of geographic information for modeling spatial dynamics in an interoperable environment. As an example of the fields that requires such information, this work focuses only on the surface hydrology domain. Over the past decade, there have been great demands in incorporating geographic information for modeling hydrologic systems [18]. This task typically requires the establishment of the right correspondence of data across databases, 
which is a problematic issue [2]. Solving this problem requires developing a data model that captures the semantics of hydrologic concepts used in disparate databases so that the right correspondence between them can be made. This paper seeks to address this issue by examining how the notions of endurance and perdurance $[6,9]$ can be used to develop a data model for such a purpose.

Data models have previously been developed for the hydrology domain, among which the Arc Hydro data model [11] comes closest to this research. The Arc Hydro data model focuses on the description of surface hydrology and hydrography, and was built on the Environmental Systems Research Institute ArcObject data model. In this work, we are not interested in a particular GIS data model, but in a more generalized and robust mechanism to organize information about surface hydrology concepts. We apply the Basic Formal Ontology (BFO) proposed by Grenon and Smith [8], which is based on the notions of endurance and perdurance and on mereology [15], as a tool to build a data model that centers around hydrologic processes in the surface hydrology domain. The data model developed is rich in surface hydrology semantics and, therefore, offers better potential for facilitating the modeling of surface hydrology phenomena.

The remainder of this paper is structured as follows: Section 2 discusses the method used to extract concepts from the surface hydrology domain. Section 3 introduces BFO as a way to encode surface hydrology concepts. Sections 4 and 5 present the results of organizing surface hydrology concepts with the notions of perdurance and endurance, respectively. Section 6 concludes the paper.

\section{Methodology}

The development of a data model is an iterative process that typically involves the following steps: determining the purpose of developing a data model, extracting concepts, defining relations between concepts, and evaluating the results [12]. In this research, a similar methodology was adapted. Specifically, the following three steps were adapted:

1. Extract knowledge and identify the ontological commitments in a hydrologic model. The results of this step are informal.

2. Assign each entity, identified in the first step, to a category defined in BFO. We first identify perdurants, then endurants following the relations between these two entity types (Section 3.2). This sequence is important in this research, because it allows us to identify entities that are absolutely essential for describing hydrologic processes.

3. Cross-check the meanings of these entities identified in the first step against the definitions of categories and the relations between categories in BFO. The crosschecks minimize any mistake in assigning a concept to a wrong category.

The knowledge and ontological commitments about the surface hydrology domain were extracted from sources including books, journal articles, model programs, and 
source code of particular hydrologic models. These sources complement each other in a way that the model programs and source code provide "proofs" or verification for the conceptual models of the hydrologic systems laid out in books and journal articles, because those programs can be tested by comparing their estimates with the actual values obtained from a real hydrologic system.

Three hydrologic models were selected with all aforementioned sources in presence, including the HEC-HMS [17], TOPMODEL [1], and BLTM [10]. HEC-HMS is a hydrologic model that can be used to predict flood stage in response to a precipitation event. TOPMODEL is a hillslope scale hydrologic model that predicts the amount of runoff of a catchment, based on the idea that the area contributing to the runoff changes with the degree of soil saturation. BLTM is a river routing model that describes the speed of a contaminant traveling to a downstream location. These models have been chosen for two reasons. First, they have been developed over an extended period of time and have undergone several refinements. The validity of those hydrologic models is thus higher than those which have not gone through such revisions. Second, they were developed using different modeling approaches for the transportation of water on the land surface. This allows us to examine various conceptualizations of the same hydrologic process.

\section{Encoding Surface Hydrology Concepts}

Surface hydrology is concerned with the flow of water and its constituents over the land surface [4]. In order to describe such phenomena, various entities are involved, such as surface runoff or a watershed. Surface runoff is a process in which water is moved from one place to another. It is an entity that unfolds through time. A watershed is typically an entity that certain hydrologic processes act upon. The hydrologic processes may change a watershed's shape or boundary. However, the watershed does not unfold through time, but remains its identity over a prolonged period of time (i.e., the watershed you see today is the same one that you see tomorrow). An entity of the first type is called an endurant, while an entity of the second type is called a perdurant.

Differentiating these two distinctive types on surface hydrologic concepts is important in the development of a data model for the following reasons. First, the entities encoded should have a one-to-one correspondence to concepts comprehended by domain experts. This avoids any ambiguity of an entity's meaning. An entity that unfolds through time should be recognized as such. The same principle applies to an entity that retains its identity through time. Second, relations between entities can be identified more easily if the entities' types (perdurant or endurant) are known. This leads to fewer errors in the development a data model. This section describes a framework that recognizes both types of entities, that is, the Basic Formal Ontology (BFO) as presented by Grenon and Smith [8], to encode concepts in surface hydrology. 


\subsection{Endurant and Perdurant}

The most basic categorial distinction between entities relates to different modes of persistence through time. Two categories of persistent entities can be distinguished: endurants and perdurants. Endurants are wholly present (i.e., all their proper parts are present) at any time at which they exist. For example, you (an endurant) are wholly present in the moment you are reading this. No part of you is missing.

Perdurants, on the other hand, are extended in time in virtue of possessing different temporal parts at different times. As opposed to endurants, they are only partially present at any time at which they exist-they evolve over time. For example, at this moment only a (tiny) part of your life (a perdurant) is present. Larger parts of your life-such as your childhood-are not present at this moment.

Since those distinctions are so fundamental, it is critical to distinguish between perdurants and endurants at the very top of the classification hierarchy. In BFO, perdurants and endurants form disjoint category trees. Endurants are divided into two major categories: independent endurants, such as catchments and channels, and dependent endurants, such as qualities like catchment area or channel sinuosity. BFO distinguishes the following kinds of independent endurants: substances, fiat parts of substances, aggregates of substances, boundaries of substances, and cavities (Figure 1).

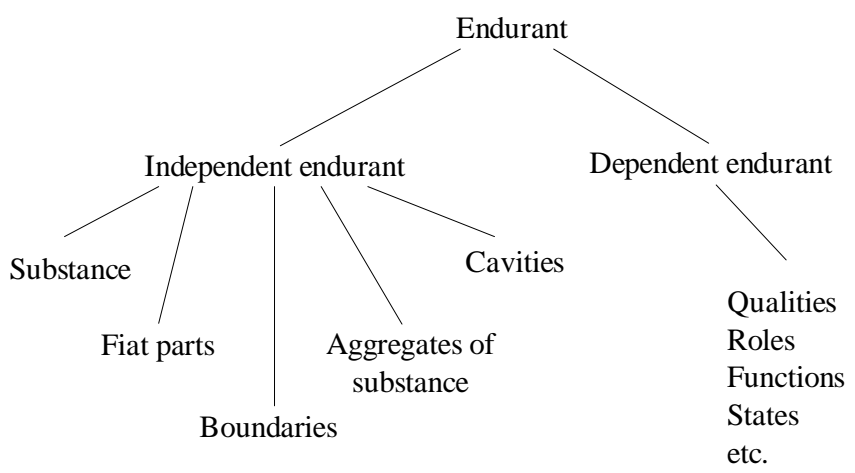

Fig. 1. Category tree of endurant (adapted from [8])

Substances are maximally connected entities. They have connected bona fide boundaries that correspond to discontinuities in the underlying reality. For example, neither a channel section nor a tributary are substances. Both are fiat parts of a channel. Fiat parts are demarked from a substance by boundaries that are not bona bide in nature but rather the result of human demarcation. Aggregates of substances are not substances either. Examples of aggregates are aggregates of several sub-catchments, channel and hillslope, etc. 
Dependent endurants are entities that cannot exist without some other entity or entities upon which they depend. Dependent endurants can include, among others, qualities, roles (a canal can play the role of a channel), functions (one function of a riverbank is to accommodate excess water), and states (a catchment is in the state with full water storage).

Perdurant, the second top-category in BFO, distinguishes processes, their fiat parts, aggregates, and boundaries (Figure 2). Precipitation is a process, as is the graduation of a channel [16]. The development of a flood plane by a channel is a fiat part of the perdurant channel gradation. The interception of precipitation can be an aggregate of canopy interception and surface interception.

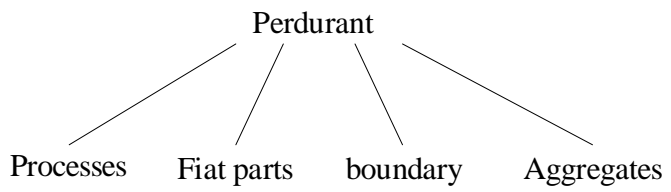

Fig. 2. Category tree of perdurant (adapted from [8])

\subsection{Cross-Categorial Relations}

Cross-categorial relations are relations that hold between the categorical distinct perdurants and endurants. The most important cross-categorial relations are participation, realization, involvement, and affecting [8]. In this paper, we mainly focus on involvement. The participation relation in our case is equivalent to the involvement relation in a reverse direction (i.e., one is the converse of the other). Involvement is a relation that holds between a perdurant and a substance endurant. Every perdurant acts upon at least one such endurant. Examples for involvement relations are a channel flow process that involves a flow plane and water, evapotranspiration that involves soil matrix and vegetation, etc. These examples can be easily translated into the participation relations: flow plane and water participates in the channel flow process.

\subsection{A Case Study - Catchment, Watershed, and Basin}

In surface hydrology the terms catchment, watershed, and basin are often used interchangeably to refer to the same entity. Results from the first step of our methodology showed that in HEC-HMS watershed and catchment are used to refer to identical entities and in TOPMODEL the same situation applies to basin and catchment. In different occasions, they may or may not carry the same meaning depending on how water flow is described. Figure 3 shows some possible meanings identified from the three hydrologic models. 


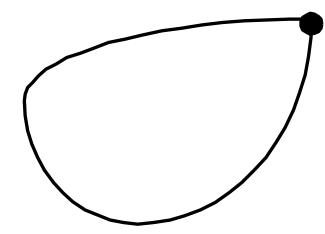

(a)

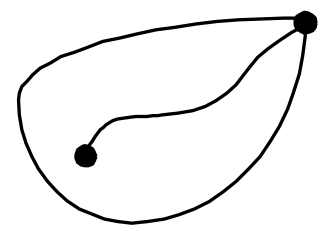

(b)

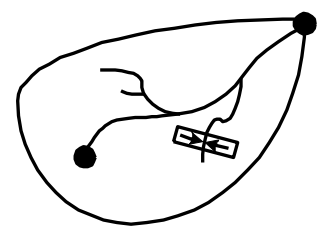

(c)

Fig. 3. Various meanings of catchment, watershed, and basin

The case (a) indicates that the catchment is represented by the outlet indicated by the black dot. The spatial variation within the catchment is not very important. In case (b) the catchment is similar to that of case (a), but now a channel (represented by the curved line in the catchment) is added into the catchment, indicating the consideration of water velocity in modeling the water flow. Case (c) is a more complex version of the case (b) in the sense that it considers a channel network instead of one channel. In addition, it also considers the hillslope flow to the channel network, as indicated by the strips on both side of the channels.

The discussion above demonstrates the need to develop a consistent data model for surface hydrology, if one needs to integrate data from disparate sources. Without such a data model it is difficult to distinguish whether the notion of catchment employed in model $\mathrm{A}$ is equivalent or similar to the notion of watershed (or basin) employed in model B. The rest of this section discusses how the notions of endurant and perdurant can provide the basis for developing a data model in surface hydrology. We first apply the notion of perdurant to classify concepts of surface hydrology. This reflects the priority of process in surface hydrology. Through the involvement (or participation) relation between perdurants and endurants, we eventually define the concept of a catchment and other concepts needed to support such a definition.

\section{Perdurants in the Surface Hydrology Domain}

In this section, we introduce entities that fall under the perdurant category. The discussion starts with processes and how they are determined. It continues with entities that are aggregates and fiat parts in the perdurant categorical tree. It ends with discussion on how involvement (or participation) relation leads to the identification of endurants.

\subsection{Processes}

Following the methodology specified in Section 2, eleven kinds of process have been identified under the scope of this research:

1. Precipitation: a process that moves water from the atmosphere to a land surface. 
2. Canopy interception: a process in which vegetation catches and stores precipitation.

3. Surface interception: a process where precipitation is caught and stored in a small surface depression such as a puddle.

4. Evapotranspiration: a process that returns water to the atmosphere.

5. Overland flow: surface runoff that occurs in the form of sheet flow over the land surface without concentrating in clearly defined channels.

6. Channel flow: water flowing in the channel.

7. Infiltration: a process of water entry at the land surface into the soil.

8. Return flow: the movement of water from soil to land surface that eventually reaches to a channel.

9. Through flow: the movement of water within unsaturated or saturated soil layers located immediately beneath the surface.

10.Percolation: the movement of water through the interstices of rock or soil.

11.Base flow: the portion of channel flow originating from underground aquifer.

These processes were obtained first by determining if they qualify as perdurants. By accepting them as processes, we are able to reason about their temporal parts. For example, it is possible to obtain water transported by evapotranspiration process of yesterday, or to describe the behavior of channel flow (e.g., how fast water travels to a down stream location). The concept of perdurance is especially helpful in cleaning up ambiguity and duplications in the following cases:

1. Endurant or Perdurant?

In the first step of our methodology, precipitation volume was identified as an input to all three hydrologic models. Precipitation volume is a quality and, according to the BFO classification, a dependent endurant. It is dependent on another endurant, such as a catchment in HEC-HMS or a hillslope in TOPMODEL. While the precipitation volume serves as an important factor in describing hydrologic behavior, we did not find any description about the precipitation process in the hydrologic models examined. None of the models addresses the important question of how the precipitation process is formed or how its intensity or location changes through time. In this research, we represent precipitation as a process because talking only about qualities of processes does not allow us to reason about when and where a process occurs. What we really need is the process itself.

2. Naming Heterogeneity between Processes

A case of naming heterogeneity was identified in the three hydrologic models. Naming heterogeneity refers to the circumstances where the semantically-alike entities are named differently [2]. Such cases can be identified through comparing meaning carried by entities of the same type, which in this case are those of process type. The case of semantic heterogeneity was found for depression storage and surface storage. Depression storage is a perdurant indicating water volume held in land surface depressions such as small ponds. Surface storage for runoff generation is a perdurant indicating water volume held in local depressions in the ground surface [17]. Given 
that depression storage and surface storage both perform the same function and occur on the same endurants, they are identified as a single process.

\section{Process Specialization}

Besides naming heterogeneity, we also found examples were one hydrologic process is a special case of another hydrologic process. Consider the processes lateral flow and source flow. Lateral inflow moves water from a flow plane into a channel. The flow plane can be on the left side or right side of a channel. On the other hand, source flow moves water from a flow plane to a source of a channel. Both types of flow move water in the form of sheet flow. The meanings of lateral inflow and source flow are essentially overland flow, except that the flow destination is explicitly defined. Both are therefore considered as two specific cases of the more general concept overland flow.

\subsection{Aggregates Perdurants}

Some processes identified from the three hydrologic models are composed of the eleven processes listed above. Examples of such aggregates are listed in Table 1.

Table 1. The list of aggregate perdurants identified from the three hydrologic models

\begin{tabular}{|l|l|}
\hline Aggregate Perdurant & Processes Included in an Aggregate Perdurant \\
\hline Loss & evapotranspiration, interception, infiltration \\
\hline Precipitation excess & precipitation, loss \\
\hline Interception & canopy interception, surface interception \\
\hline Runoff generation & precipitation, loss, overland flow, channel flow \\
\hline Direct runoff & $\begin{array}{l}\text { precipitation, overland flow, through flow, infiltration, } \\
\text { return flow }\end{array}$ \\
\hline
\end{tabular}

To see the usefulness of the notion of aggregates of processes consider the notion of direct runoff. It can be understood as the composition of overland runoff and through flow [17] of a catchment and thus is an aggregate formed by multiple perdurants. According to the analysis in Section 4.1, overland flow takes place on the land surface and through flow occurs in the soil layer immediately beneath the land surface. Due to the aggregation of these two processes, the resulting aggregate process, direct runoff, includes the amount of water flowing on the land surface and immediately below the land surface. Aggregation of those two hydrologic processes causes us to consider two other hydrologic processes occur in the same spatial region, namely infiltration and return flow, to be included for a complete description of direct runoff. The notion of aggregate endurant thus helped us to find and revise an incomplete definition of a hydrologic process in the models we analyzed.

Aggregates can also help identify semantically similar concepts. In a sub-model (i.e., Kinematic Wave Model) of HEC-HMS that describes overland flow, lateral flow is an aggregate of precipitation and loss. It is therefore identical to precipitation excess as defined in Table 1. The concept of lateral inflow in this sense was dropped. 


\subsection{Fiat parts}

The processes identified in Section 4.1 can have arbitrary temporal parts or parts that are based on the endurant parts. The first three hours of a precipitation event is an example of arbitrary temporal parts. From the three hydrologic models examined in this research, tributary flow was identified as a fiat part that is based on the endurant parts. Tributary flow is the movement of water in a tributary. The tributary can be defined arbitrarily depending on the modeling needs, but its existence depends on a channel. If a tributary (an endurant) is part of a channel (defined in 5.5), and if tributary flow is governed by the same physical laws as that of channel flow, then tributary flow can be considered a fiat part of the channel flow. Tributary flow in the three hydrologic models that we examined always exhibited these characteristics. It was, therefore considered a fiat part of a channel flow, and was merged with the channel flow concept.

All perdurant entities identified above are shown in Figure 4.

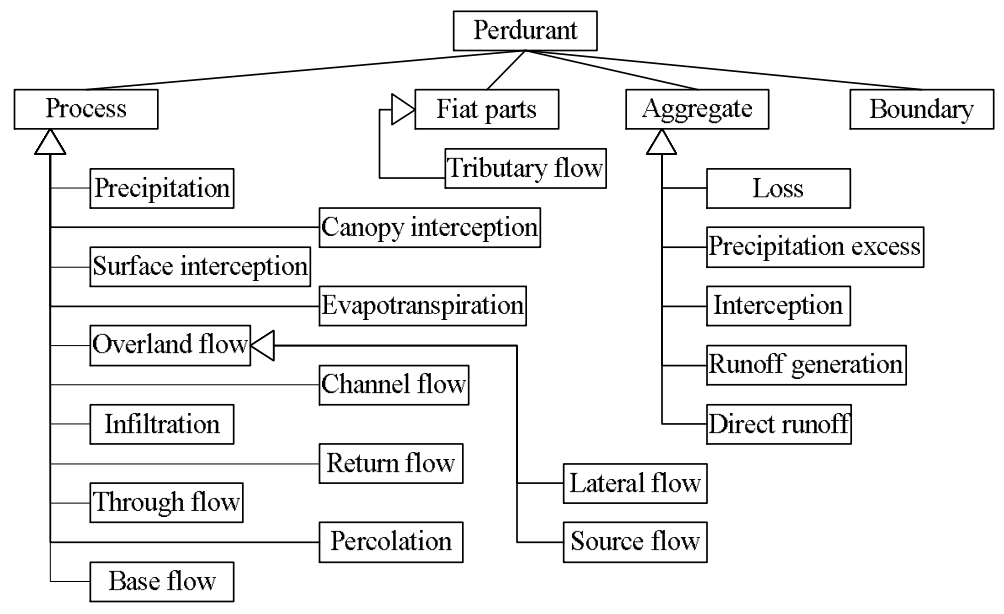

Fig. 4. Perdurants in the Surface Hydrology Domain (Triangle indicates is-a relation)

\subsection{Involvement between Perdurants and Endurants}

Perdurants can have certain relations with endurants. For example, in order to describe the evapotranspiration process in TOPMODEL, three variables are needed: root zone storage, maximum root zone storage, and potential evapotranspiration rate. All three variables are qualities of a catchment (defined in 5.7). In addition to the catchment, sufficient amount of water is needed so that evapotranspiration can take place. The evapotranspiration process thus involves a catchment and water (defined in 5.1). 
By tracking all variables needed to describe the hydrologic processes mentioned in this Section, additional independent endurants that possess these qualities were identified and are listed in Section 5. Dependent endurants are not included in this discussion for the purpose of clarity.

\section{Endurants in the Surface Hydrology Domain}

In this section, we will discuss eight independent endurants (substances) that were identified following the methodology set forth in Section 2. These independent endurants are entities that are wholly present at any time at which they exist. For each kind of endurant, the definition and its participation relation to the kinds of perdurants that were identified in Section 4 will be discussed.

\subsection{Water}

Water is an entity that is moved between all other endurants mentioned in this section. It may lose parts (water on the land surface may evaporate), or its acidity may change. Nonetheless the water still remains the same entity. It is therefore an endurant. Water can be moved around by different perdurants mentioned in Section 4, and thus participates in all perdurants listed in that section.

In the BLTM model, water is modeled by a concept called a parcel. A parcel has a pre-defined water volume and is treated as an object that travels along a channel. In this work, parcel was not included mainly because it is an artificial construct introduced for the purpose of reducing computational complexity.

\subsection{Sink}

A sink is a substance endurant that exhausts the water from either a channel (defined in 5.5) or a catchment (defined in 5.7). A sink can participate in two types of process: source flow (a type of overland flow) or channel flow. For source flow, water running on a flow plane converges at the sink. For channel flow, water traveling in a channel drains out of the channel at its sink.

\subsection{Source}

A source contributes water to a receiving endurant, which is normally a channel (defined in 5.5) or a catchment (defined in 5.7). It can participate in channel flow, where source provides input water volume to the channel. It can also participate in overland flow (in the form of a spring) to provide water input in a flow plane. 


\subsection{Junction}

A junction connects at least two channel parts, such as a tributary and a channel section. It participates in channel flow in the sense that water flow from two channel sections is merged or flow from one channel section underwent bifurcation.

\subsection{Channel}

A channel is a linear feature connecting at least one source to a sink. A channel participates in channel flows, return flow, and base flow. With these flows, the direction of a channel can be defined as the flow of water from the source to the sink. If the channel has branches, the channel will also have junctions at the location where the channel and the branches meet. A channel is associated with several flow planes (defined in 5.6). Water on those flow planes flows into the channel.

A channel can have parts, which are themselves channels. Examples of those channel parts include sub-channels, tributaries, branches, or any channels of a lower order. The channel order designates the relative position of a sub-channel in a channel. A sub-channel without a tributary is a first-order channel. The convergence of two first-order sub-channels produces a second-order channel; the convergence of two second-order sub-channels produces a third-order channel. These channel parts participate in channel flow in the sense that they participate in channel flow parts, such as tributary participates in tributary flow indicated in 4.3.

The conditions for a channel $y$ to be a sub-channel of another channel $x$ are as follows:

- Channel $y$ is part of channel $x$.

- The sink of channel $y$ is a junction or a sink of channel $x$.

- The water flows from channel $y$ to channel $x$.

If the sub-channel is a channel section, an addition rule applies:

- It has only one source and one sink.

\subsection{Flow plane}

A flow plane is an area that contributes water to a channel. It participates in processes including precipitation, surface interception, overland flow (or lateral inflow), infiltration, and aggregate perdurants including precipitation excess, direct runoff, and loss. The flow plane itself does not have any channel, given that only sheet flow exists on a flow plane.

Depending on the specific relationship with a channel, four types of flow planes can be identified: left plane, right plane, source area, and inter-catchment area. The left flow plane contributes flow to a channel from its left side. The side of the channel is defined in terms of the channel flow direction. The right flow plane contributes flow to a channel from its right side. The source area contributes flow to the source of a channel. The last type of flow plane, the inter-catchment area [14], contributes flow to particular channels. Two conditions must hold for the inter-catchment area: 
1. Those channels have to be at least of channel order 2. This means that there exist some channels of order 1 merging at junctions.

2. The inter-catchment area is within a catchment that encompasses those channels.

\subsection{Catchment}

A catchment is an areal feature that is defined by an outlet, the channel that drains to the outlet, and the area that drains to the channel. It participates in precipitation, surface interception, evapotranspiration, overland flow, channel flow, baseflow, infiltration, as well as in aggregate perdurants including direct runoff and interception.

Similar to the channel, a catchment can also be designated a catchment order. The order is defined as the largest channel order contained in the catchment. A catchment's boundary is defined as the area upstream of the outlet that, with the presence of water, drains to the outlet. Within this area, a catchment has flow planes and a channel. A catchment of order 1 has only a left flow plane, a right flow plane, and its source area. A catchment of order higher than 1 has all four types of flow planes. A catchment also includes earthy material, such as soil and rock, as well as land cover, such as tree and grass. The catchment may contain or carry fluid (e.g., water), but the fluid is not a part of it.

A catchment can have sub-catchments, as long as each sub-catchment is homogeneous in certain catchment-dependent entities, such as the intensity of the precipitation or the soil's infiltration rate. A sub-catchment has a sink and it has its own channel. In this respect, a sub-catchment is a catchment if the catchment met the characteristic of homogeneous property. The conditions for a catchment $y$ to be a sub-catchment of another catchment $x$ are as follows:

- The earthy material of the catchment $y$ is part of that of the catchment $x$.

- The channel of the catchment $y$ is part of that of the catchment $x$.

- The outlet of the catchment $y$ is a junction of the channel of catchment $x$

\subsection{Atmosphere}

Atmosphere is the gaseous envelope of a celestial body. It participates in precipitation and evapotranspiration.

All endurant entities identified above are shown in Figure 5. 


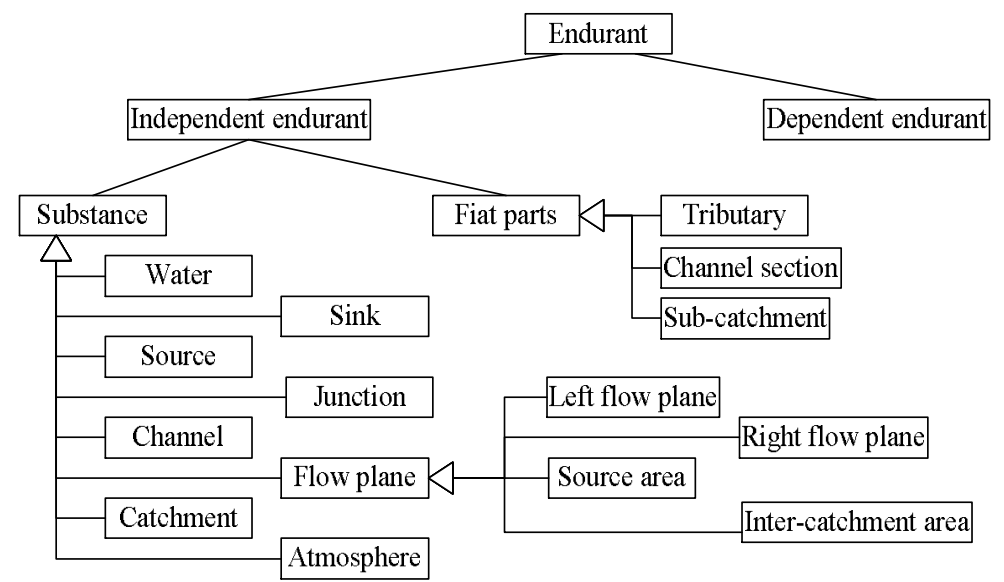

Fig. 5. Endurants in the Surface Hydrology Domain (Triangle indicates is-a relation)

\section{Discussion and conclusions}

In this paper, we demonstrated how the notions of endurance and perdurance can help us to develop a data model focusing on processes in surface hydrology. We incorporated the following notions in our data model: substance endurants, fiat parts, aggregates, and dependent endurants to identify endurants such as catchment and channel, as well as process, fiat parts, and aggregates to identify perdurants such as precipitation and overland flow. In Section 4 and 5 we identified entities that are indispensable to understanding the movement of water on the surface of the earth. We showed how BFO can be used not only as a tool to assist in capturing concepts for surface hydrology, but also in identifying missing, duplication, or specialized concepts. The use of the endurance and perdurance notions thus contributes to a better understanding of hydrologic concepts, in the sense that they are now more structured and less ambiguous. The data model proposed in this paper is more consistent than those obtained in step 1 listed in Section 2. It is more consistent because redundant entities were either removed (e.g., a river or a reach as to a channel) or merged through parthood relations (e.g., tributary, sub-reach as part of a channel), artificial entities introduced for the purpose of computational simplicity were removed, and several missing entities were identified. It is possible to know what a process is by examining its variables encoded as dependent endurants. Through the participate relation between endurants and perdurants, it is possible to identify the functions of an endurant in monitoring water flow on the land surface.

One interesting issue that is not handled in this work is the issue of granularity [3]. Some dependent endurants identified for catchment, such as the two routing coefficients in a sub-model of HEC-HMS, indicate the intention of describing overland flow and channel flow behavior in the model. However, the substance endurants 
involved do not include a channel or a flow plane of any sort. This may suggest that it is necessary to introduce the concept of granularity in the development of a data model so that it more completely describes surface hydrology.

Future works will need to provide a formalization of the presented data model using formal tools like predicate logic, and to verify the completeness of the data model. The completeness of the data model can be verified by using Haskell, as suggested by Winter and Nittel [19]. We also wish to broaden the scope of this research to the ground water domain.

\section{Acknowledgment}

Support for the second author from the Wolfgang Paul Program of the Alexander von Humboldt Foundation and from the National Science Foundation Research Grant BCS-9975557: Geographic Categories: An Ontological Investigation, is gratefully acknowledged. Partial Funding for the third author was provided by Grant \# R82-7961 from the U.S. Environmental Protection Agency's Science to Achieve Results (STAR) program. This paper has not been subjected to any EPA review and therefore does not necessarily reflect the views of the Agency, and no official endorsement should be inferred. The authors also acknowledge the helpful suggestions of the anonymous reviewers of this paper.

\section{References}

1. Beven, K.J. and Kirkby, M.J.: A Physically Based, Variable Contributing Area Model of Basin Hydrology. Hydrological Sciences. 24 (1979) 43-68

2. Bishr, Y.: Overcoming the Semantic and Other Barriers to GIS Interoperability. International Journal of Geographical Information Science. 12(4) (1998) 299-314

3. Bittner, T. and Smith, B.: A Theory of Granular Partitions. In Duckham, M., Goodchild, M.F., and Worboy, M.F. (ed.): Foundations of Geographic Information Science. Taylor \& Francis, London. (2003) 117-151

4. Chow, V.T., Maidment, D.R., and Mays, L.W.: Applied Hydrology. McGrawHills Inc, New York (1988)

5. Feng, C.-C. and Flewelling, D.M.: Assessment of Semantic Similarity between Land Use/Land Cover Classification Systems. Computers, Environment, and Urban Systems. 28(3) (2004) 229-246

6. Gangemi, A., Guarino, N., Masolo, C., Oltramari, A., and Schneider, L.: Sweetening Ontologies with DOLCE. AI Magazine. 24(3) (2003) 13-24

7. Goodchild, M.F.: Communicating Geographic information in Digital Age. Annals of the Association of American Geographers. 90(2) (2000) 344-355

8. Grenon, P. and Smith, B.: SNAP and SPAN: Towards Dynamic Spatial Ontology. Spatial Cognition and Computation. 4(1) (2004) 69-104

9. Hawley, K.: How things persist. Clarendon Press, Oxford (2001)

10. Jobson, H.E. and Schoellhamer, D.H.: Users Manual for A Branched Lagrangian Transport Model. United States Geological Survey: Reston, Virginia. (1987)

11. Maidment, D.R.: Arc Hydro: GIS for Water Resources. ESRI Press, Redlands (2002) 
12. Noy, N.F. and McGuinness, D.L.: Ontology Development 101: A Guide to Creating Your First Ontology.

http://protege.stanford.edu/publications/ontology_development/ontology101.pdf, (2001)

13. Rodríguez, A.M. and Egenhofer, M.J.: Determining Semantic Similarity among Entity Classes from Different Ontologies. IEEE Transactions on Knowledge and Data Engineering. 15(2) (2003) 442-456

14. Schumm, S.A.: Evolution of Drainage Systems and Slopes in Badlands at Perth Amboy, New Jersey. Department of Geology, Columbia University: New York, New York. (1954)

15. Simons, P.: Parts, A Study in Ontology. Oxford University Press, Oxford (2000)

16. Strahler, A. and Strahler, A.: Introducing Physical Geography. John Wiley and Sons, Inc., New York (2003)

17. USACE: Hydrologic modeling system HEC-HMS, Technical reference manual. US Army Corps of Engineers, Hydrologic modeling center, HEC: Davis, CA. (2000)

18. Westervelt, J. and Shapiro, M.: Combining Scientific Models into Management Models, in GIS/EM4: Banff, Canada. (2000)

19. Winter, S. and Nittel, S.: Formal Information Modelling for Standardization in the Spatial Domain. International Journal of Geographical Information Science. 17(8) (2003) 721-741 